Temporal arteritis). Significant vascular FDG involvement was detected $(77.7 \%)$ in these patients. 4 of 7 patients diagnosed Takayasu arteritis were not seen any pathological finding in the other imaging modalities. Vascular FDG uptake $(p<0.001)$ was statistical significantly higher in the patients diagnosed with rheumatic disease whereas lymph node $(p=$ 0.013 ) and bone ( $p=0.005)$ FDG uptake were significantly higher in patients with malignancy. Patients with malignancy had the highest SUVmax mean value $(p<0.001)$.

Conclusion: Our findings reveal that PET-CT is a useful imaging modality in the FUO/IUO patients. Inflammatory diseases, especially large vessel vasculitis, should be considered in addition to malignancies in these patients, and subclinical inflammation in the blood vessel can be visualised by PET -CT

Disclosure of Interests: None declared

DOI: 10.1136/annrheumdis-2019-eular.7085

\section{AB1190 EXTENDED POLY-DIMENSIONAL IMMUNOME CHARACTERIZATION (EPIC): A WEB-BASED IMMUNE REFERENCE ATLAS OF THE HEALTHY HUMAN IMMUNOME AND A TOOL FOR TRANSLATIONAL MEDICINE}

Joo Guan Yeo ${ }^{1,2}$, Lu Pan ${ }^{1}$, Martin Wasser ${ }^{1}$, Pavanish Kumar ${ }^{1}$, Thaschawee Arkachaisri²,3, Su LI Poh ${ }^{1}$, Fauziah Ally ${ }^{1}$, Jing Yao Leong ${ }^{1}$, Kee Thai Yeo ${ }^{1,2}$, Liyun Lai ${ }^{1}$, Angela Yun June Tan ${ }^{2}$, Salvatore Albani ${ }^{1,2}$. ${ }^{1}$ Translational Immunology Institute, SingHealth/Duke-NUS Academic Medical Centre, Singapore, Singapore; ${ }^{2}$ KK Women's and Children's Hospital, Singapore, Singapore; ${ }^{3}$ Duke-NUS Medical School, Singapore, Singapore

Background: An atlas of the developing immune system will not only improve our understanding of normal immune ontogenesis but more importantly, aid in our identification of disease-associated cell subsets. However, such a resource is still unavailable despite accessibility to technologies like mass cytometry due to the general focus on specific cell subsets or ages. There is a critical unmet need for standardized datasets depicting at single cell level and with high dimensionality the entire developmental gradient of the healthy immune system from the neonatal to adult age.

Objectives: We aim to provide a detailed depiction of the architecture of the human healthy Immunome across an entire age gradient.

Methods: We have created a high dimensional atlas of the healthy human immunome (EPIC: Extended Poly-dimensional Immunome Characterization) by interrogating the peripheral blood mononuclear cells (PBMC) of over 200 healthy subjects, ranging from cord blood to adult age, with 63 unique mechanistic and phenotypic markers per cell by mass cytometry (CyTOF). The EPIC analytical and visualization pipeline is based on an open source web-based $R$ Shiny bioinformatics toolkit that allows it to be easily accessible to the research community.

Results: EPIC can be mined in various ways, for instance to follow developmental changes of any given cell subset or to depict the architecture of the Immunome at any given age range. For example, transition developmental milestones were observed in the TNF $\alpha+$ CD4+ T cells where the size of its memory subset would exceed its naive subset at 8 year old. There was a significant reduction and increase in the frequency of the naive and memory TNF $\alpha+$ CD4+ T cells with a Spearman's correlation coefficient, rho, of -0.4662 and 0.4164 respectively. More importantly, we have built and will keep developing datasets from various immune mediated diseases using the same approach. Consequently, by providing the healthy standard, EPIC enables the depiction and dissection of disease-dependent perturbations of the Immunome architecture.

Conclusion: EPIC provides a transformational conceptual advance in Translational Immunology from individual subset focused to immune architecture based approach for the understating of physiology and pathogenesis of immune mediated mechanisms. We intend to make EPIC available to the entire community in its full capacity.

\section{REFERENCES}

None

Disclosure of Interests: Joo Guan Yeo: None declared, Lu Pan: None declared, Martin Wasser: None declared, Pavanish Kumar: None declared, Thaschawee Arkachaisri Speakers bureau: Abbvie Pte, Ltd, Su Li Poh: None declared, Fauziah Ally: None declared, Jing yao Leong: None declared, Kee Thai Yeo: None declared, Liyun Lai: None declared, Angela Yun June Tan: None declared, Salvatore Albani: None declared DOI: 10.1136/annrheumdis-2019-eular.4557

\section{$\mathrm{AB} 1191$ \\ DIFFERENTIAL DIAGNOSIS OF SERONEGATIVE ARTHRITIS: DIAGNOSIS ONE YEAR FOLLOW UP AFTER FIRST DIAGNOSIS}

Ichiro Yoshii ${ }^{1}$, Tatsumi Chijiwa ${ }^{2}$, Naoya Sawada ${ }^{3}{ }^{1}$ Yoshii Hospital, Rheumatology and Musculoskeletal Medicine, Shimanto City, Japan; ${ }^{2}$ Kochi Memorial Hospital, Rheumatology, Kochi, Japan; ${ }^{3}$ Dohgo Onsen Hospital, Rheumatology, MAtsuyama, Japan

Background: The ACR/EULAR classification criteria (AECC) for rheumatoid arthritis (RA) is well known criteria for early diagnosis of RA with high sensitivity. However, the criteria weigh three points for seropositive, thus automatically subtracted 3 points for seronegative arthritis. Recently elderly onset RA is increasing, that has relatively higher ratio of seronegative than young onset RA.

Objectives: To compare diagnosis of seronegative arthritis between initial and second diagnosis after one year follow up.

Methods: Arthritis patient who are sustained for more than one year since first refer despite his/her ACPA or Rheumatoid Factor were negative, were collected. From these patients, difference from initial diagnosis to second after one year follow up was evaluated statistically with chi square test. Mean EACC score and mean involved joint count for large and small joint (LJC and SJC) at initial diagnosis were compared with each of second diagnosis statistically with Mann Whitney $U$ test (MWU). Clinical course evaluated with 28-joints disease activity score (DAS28) and Health Assessment Questionnaire Disability Index (HAQ-DI) for each diagnosis was compared at every other 3 months with MWU. Comparison between 475 seropositive RA patients treated in the same institute and seronegative RA in the study was also evaluated in a same manner.

These patients' sensitivity and specificity (Sens \& Specs) in according with 1987 ACR diagnosis criteria (1987ACR) was also evaluated and compared with Sens \& Specs of AECC.

Results: Ninety-six patients were enrolled. In these, RA was diagnosed to 18 patients and 78 were unclassified arthritis as first diagnosis. Second diagnosis of these patients were RA for 41, spondyloarthritis (SpA) for 22 , other collagen disease (CD) for 5, osteoarthritis $(O A)$ for 9 , hypothyroidism (HTh) for 2, non-tuberculotic mycobacterium (NTM) for 1, and unclassified arthritis (UA) for 16. Mean EACC score and range for each second diagnosis was 5.2 and 2 to $7,4.4$ and 3 to $7,4.4$ and 3 to 5 5.0 and 4 to $6,4.0$ and $4,4.0$, and 4.25 and 3 to 5 , for RA, SpA, CD, OA, HTh, NTM, and UA, respectively. There is no significant difference between any pair of second diagnoses. LJC and SJC of each second diagnosis was 3.2 and 9.8, 5.7 and 4.8, 2.4 and 4.8, 2.4 and 8.1, 2.0 and 6,2 and 2, and 1.8 and 5.9, for RA, SpA, CD, OA, HTh, NTM, and $U A$, respectively. There is also no significant difference between any pair of second diagnoses (Table).

In clinical course, there is no significant difference between the second diagnoses, and also no significant difference between seropositive and seronegative RA.

In these seronegative arthritis patients, Sens \& Specs of RA in accordance with $1987 \mathrm{ACR}$ were $75.0 \%$ and $43.4 \%$, while $83.3 \%$ and $66.7 \%$ in accordance with AECC.

Conclusion: Diagnosis of seronegative RA is not uncomplicated, whereas rheumatologist's diagnostic skill is questioned.

Table: Comparison between second diagnosis

\begin{tabular}{lcccccc}
\hline $\begin{array}{l}\text { Second } \\
\text { diagnosis }\end{array}$ & First diagnosis & $\mathrm{N}$ & AECC & $\begin{array}{l}\text { Joint } \\
\text { point }\end{array}$ & LJC & SJC \\
\hline RA & RA(15), UA(26) & 41 & 5.2 & 3.7 & 3.2 & 9.8 \\
SpA & RA(1), UA(21) & 22 & 4.4 & 3.0 & 1.9 & 5.7 \\
CD & UA(5) & 5 & 4.4 & 2.8 & 2.4 & 4.8 \\
OA & RA(2) OA or UA & 9 & 5.0 & 3.4 & 2.4 & 8.1 \\
& $(7)$ & & & & & \\
HTh & UA(2) & 2 & 4.0 & 3.0 & 2.0 & 6.0 \\
NTM & UA(1) & 1 & 4.0 & 2.0 & 2.0 & 2.0 \\
UA & UA(16) & 16 & 4.25 & 3.0 & 1.8 & 5.9 \\
\hline
\end{tabular}

Disclosure of Interests: None declared

DOI: 10.1136/annrheumdis-2019-eular.913 